\title{
Progression of diabetes, ischemic heart disease, and chronic kidney disease in a three chronic conditions multistate model
}

\author{
Chathura Siriwardhana*, Eunjung Lim, James Davis and John J. Chen
}

\begin{abstract}
Background: Diabetes mellitus, ischemic heart disease, and chronic kidney disease are three major chronic conditions that develop with increasing risks among adults as they get older. The interconnectedness of these three chronic conditions is well known, while each condition acts as a prognostic risk factor for the other two. It is important to understand the progressive relationships of these three conditions over time in terms of transitioning between clinical states and the impact on patients' survival.
\end{abstract}

Methods: We investigate the survival characteristics of a Medicare population aged 65 years and above in a multistate system that contained clinical states specified by death and diagnosis combinations of three chronic conditions. The study was conducted using Hawaii Medicare claims data from 2009 to 2013. To evaluate the progression of a subject with one of the newly diagnosed chronic conditions, we analyzed quantities such as state occupation probabilities in eight states and hazards of sixteen transition types. We quantified effects and significances of potential covariates such as age, gender, race/ethnicity, comorbidity burden and financial status on these temporal functions. Nonparametric method of estimating state occupation probabilities and pseudo-value based method for estimating covariate effects of a survival system were utilized.

Results: We found a range of age, gender, race/ethnicity and financial status based interesting covariate influences on transitions and state occupation probabilities of the system.

Conclusion: Survival characteristics of the disease system are influenced by subject-specific effects. Subgroup-specific interventions/screenings should be considered for the optimal prevention and care.

Keywords: Aging, Chronic kidney disease, Diabetes, Ischemic heart disease, Medicare data, Multistate model

\section{Background}

Diabetes mellitus (DM), ischemic heart disease (IHD), and chronic kidney disease (CKD) are three major chronic conditions that develop with increasing risks among adults as they get older [1-3]. In recent years, approximately $31.0,25.9$, and $4.4 \%$ of the elderly US population aged 65 years and above suffered from DM, IHD, and CKD, respectively. These three chronic conditions are associated with high health care expenses [4-6]. In 2013, the total health care costs for DM, IHD, and CKD were 101.4, 88.1, and 13.5 billion dollars, and the elderly population accounted for $42.8,61.2,52.5 \%$ of these costs, respectively [7].

\footnotetext{
* Correspondence: cksiri@hawaii.edu

Biostatistics Core, Department of Complementary and Integrative Medicine, University of Hawaii John A. Burns School of Medicine, Biosciences Building, Suite 211, 651 Ilalo Street, Honolulu, HI 96813, USA
}

The interconnectedness of these three chronic conditions is well known, while each condition acts as a prognostic risk factor for the other two. Previous studies discuss risks associated between pairs of these conditions and their subtypes on lifetime, quality of life, and etiological aspects. For example, a diagnosis of CKD not only increases the risk of cardiovascular morbidity but worsens cardiovascular condition outcomes if a subject has both diseases together [8]. Some evidence suggests that around half of all heart failure patients suffer at least some level of CKD in their lifetime [9]. Accordingly, patients with CKD are strongly recommended to have regular screenings for the presence of cardiovascular related complications for early intervention. Cardiovascular complications also play a critical role in the development of CKD [10,11], and evidence shows that CKD prevalence is high among the population with

(c) The Author(s). 2018 Open Access This article is distributed under the terms of the Creative Commons Attribution 4.0 International License (http://creativecommons.org/licenses/by/4.0/), which permits unrestricted use, distribution, and 
undiagnosed diabetes and pre-diabetes [12]. In addition, DM is reported as a major risk factor for cardiovascular disease [13] and a leading risk factor for end-stage renal disease, co-occurring in half of the patients with CKD $[14,15]$.

Generally, these three conditions do not necessarily follow a generic progression pattern, but one condition or pairs of them act as a possible risk factor for developing the other conditions. Therefore, it is important to understand the progressive relationships of these three conditions over time in terms of transitioning between clinical states and the impact on patients' survival, especially among the elderly, who are at increased risk for these conditions. To our knowledge, there is no published literature discussing these conditions together with respect to state transitioning and patients' survival. To fill this gap, we investigated the characteristics of a progressive multistate system structured with these three major chronic conditions for elderly individuals using Hawaii Medicare data.

The main objectives of this study are two-fold: (1) to determine overall survival characteristics associated with DM, IHD, and CKD conditions in adults aged 65 years and above as related to transitions between various clinical stages; and (2) to demonstrate the use of health insurance data to evaluate the survival characteristics of a chronic disease system.

\section{Methods}

\section{Data}

We employed a retrospective cohort analysis of 23,030 individuals aged 65 years or above who experienced at least one of the following conditions: DM, IHD, and CKD, from January 1, 2009 through December 31, 2013 using Hawaii Medicare claims data. This data allowed us to systematically track the occurrence of a set of selected disease conditions for up to five years and provided covariate information such as demographic data and the presence of other co-morbidities. Data on both inpatient and outpatient visits were utilized. The International Classification of Disease 9th Revision (ICD-9) diagnosis codes [16] were used for specifying the disease conditions. Specifically, the series of ICD-9 codes of 250, 410414, and 585 were used to identify DM, IHD, and CKD, respectively.

The Medicare master beneficiary summary file was used to identify gender and race/ethnicity. Race/ethnicity was categorized as White, Asian, Native Hawaiian and Pacific Islander (NHPI), and Other group, which contained American Indian/Alaska Native, AfricanAmerican, Hispanic and unknown races that represented as a small proportion. Respective percentages of these groups were White, 28.4\%; Asian, 26.9\%; NHPI, 24.0\%; and Other, 20.7\%. In addition, we used the Charlson
Comorbidity Index (CCI) calculated at baseline that gives a one-year survival score for a patient based on chronic commodities [17]. Furthermore, we used the dual eligibility status of an individual who received both Medicare and Medicaid benefits as an indicator for socioeconomic status as it is common among individuals with low incomes. The research protocol was approved by the Institutional Review Board at the University of Hawaii.

\section{Multistate model and statistical analyses Multistate model}

In this study, we used the acyclic multistate model shown in Fig. 1 to define an interconnected progressive chronic disease system for the elderly population. In the system, there are eight clinical states an individual can occupy at a given time point. An individual starts from one of the single disease states (i.e., DM, IHD, or CKD) and moves towards the absorbing state Death either directly or through four different intermediate multi-disease states. Depending on the initial state of the individual, he/she may move to one of three dyad states: $\mathrm{DM}+\mathrm{IHD}, \mathrm{DM}+\mathrm{CKD}, \mathrm{IHD}+\mathrm{CKD}$, or to the Death state. If an individual moves to one of the dyad states, he/she may transfer to DM + IHD + CKD, or to the Death state. We controlled the complexity of the system by limiting the initial states of an individual to be one of $\mathrm{DM}$, IHD, or CKD only, and by not allowing direct transitions from states DM, IHD, and CKD directly to the $\mathrm{DM}+\mathrm{IHD}+\mathrm{CKD}$ state.

We determined the earliest date of the first disease occurrence for each individual and tracked all subsequent states along with state transition times. Starting from this point, we extracted the claims data for the three conditions to track the progression of each individual in various clinical states as indicated in Fig. 1. Here, individuals entered the system at different time points, resulting in right-censoring in the multistate model. A summary of transition counts for the system is provided in Additional file 1: Table S1 in matrix form.

\section{State occupation probabilities and transition hazards}

State occupation probabilities and state-to-state transition hazards are two important quantities for a multistate model. We used the Aalen and Johansen [18] estimator to estimate marginal state occupational probabilities, which is a nonparametric technique that provides great flexibility in handling a complex multistate system without strict model assumptions. To identify the important covariates affecting state occupation probabilities and state-to-state transition hazards, we employed the pseudo-values methods proposed by Andersen and Klein [19]. This was implemented using the generalized linear model with a logit link function to 


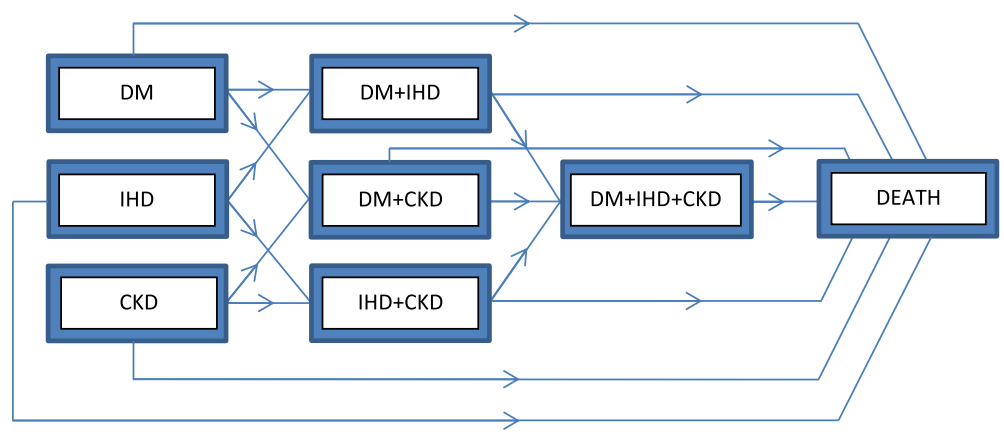

Fig. 1 Graphical representation of the progressive chronic disease network consisting of eight states

estimate the covariate effect on the state occupation while incorporating $\log$ link function for state-to-state cumulative transition hazards. Details of the aforementioned estimation procedures for a multistate model are described in the Additional file 1.

Due to the limited follow-up period the observed transitions subjected to right-censoring. The estimation was based on the assumption that individuals were randomly censored. Ninety-five percent point-wise confidence intervals of state occupational probabilities and stateto-state transition hazards were estimated using the bootstrap method with 1000 bootstrap samples. All the analyses were conducted using R-3.3.1 software, with algorithms developed incorporating existing functions in R-base packages.

\section{Results}

We evaluated the progression of a newly diagnosed subject with DM, IHD, and CKD conditions with respect to time, analyzing the state occupation probabilities of eight different states, and the hazards of sixteen transition types (see Fig. 1). We also reported effects of several important covariates, such as age, gender, race/ethnicity, comorbidity burden and financial status, on these temporal functions.

\section{Transition counts}

At baseline, individuals entered the system initiating from 9628 DM, 7943 IHD, and 5359 CKD states. Correspondingly, 6868 (71.3\%), 5276 (66.4\%), and 3295 $(61.4 \%)$ of these individuals remained in their initial states by the end of the study period. We summarized the transition counts for the study in Additional file 1: Table S1 to illustrate the basic transition characteristics for the system. For example, the cell (DM, DM + IHD) shows that of the 9628 who initiated from DM state, 1163 individuals transferred to DM + IHD state. The cell (DM + IHD + CKD, Death) indicates that out of 950 individuals who transferred to DM + IHD + CKD state, 363 individuals transferred to Death state.

\section{Marginal estimates of state occupation probability}

The initial probabilities of entering the system from DM, IHD, and, CKD clinical states were 41.9, 34.7, and 23.4\%. The probabilities of staying in these states gradually declined over time down to 27.3, 20.6, and $12.7 \%$ four years later. CKD showed the highest relative decline in the state occupational probability, resulting in the tail probability closer to half of its initial value. In Fig. 2, we provided a graphical illustration of the marginal state occupation probabilities that estimated via the Aalen-Johansen estimator along with 95\% bootstrap based point-wise confidence intervals.

After the initial state, the next three subsequent intermediate states are three dyads: DM + IHD, DM + CKD, and IHD + CKD. For these dyad sets, the state occupation probabilities increase over time with a nonlinear trend, such that they increase rapidly during the initial phase. At a later phase the degree of increment slowly dissipates, resulting in approximately 8.7, 7.0, and $5.7 \%$ for occupying DM + IHD, DM + CKD, and IHD + CKD after four years of the initial occurrence, respectively.

The occupation probability of the DM + IHD + CKD state increases at a much slower rate compared to the dyad state sets reaching a maximum value of $3.0 \%$ around 1415 days, and then declining at a very slow pace. Clearly, the intensities of state occupation probabilities for intermediate states at any given time are relatively lower than those of the initial states.

For the absorbing state of Death, the occupation probability increases rapidly at the beginning and then follows an almost linear trend with respect to time. After four years, the probability of experiencing death reaches approximately $15.1 \%$.

\section{Marginal estimates of cumulative transition hazards}

Figure 3 presents the marginally estimated state-to-state cumulative transition hazards from one given state to another by the Nelson-Aalen type hazard estimator. The details of $95 \%$ bootstrap based point-wise confidence 

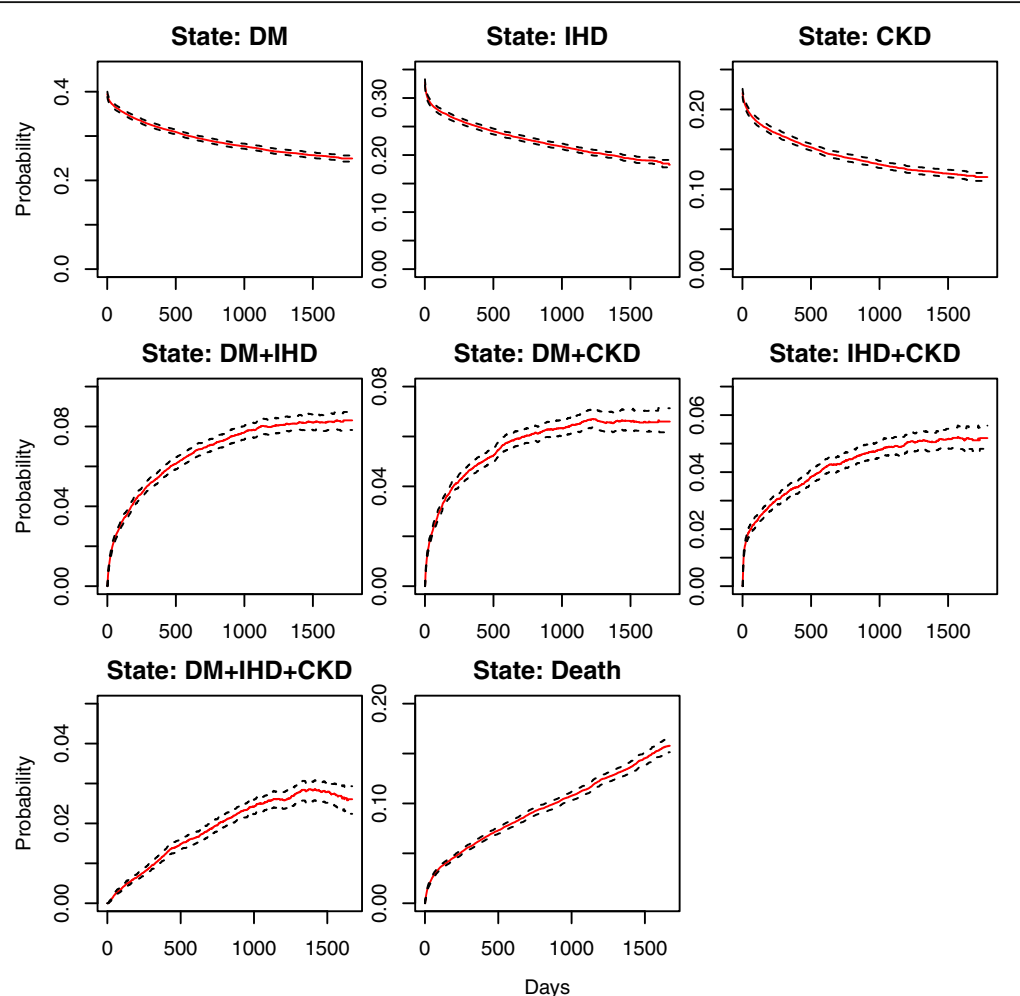

Fig. 2 Estimated marginal state occupational probabilities for the multistate model along with 95\% bootstrap based confidence bands. We illustrate state occupation probabilities with a solid line and corresponding 95\% confidence bands with dotted lines. Note: different spans of y axis were used for different sets of states

intervals of the estimated state-to-state cumulative transition can be found in Additional file 1: Figures S1-S7.

Among all the initial single disease states, subjects with DM were least likely to die at any given time. On the other hand, subjects with CKD had the highest risk of death. The corresponding death risk for CKD subjects appears to be similar to their transition hazards to any subsequent dyad states. For a subject with IHD, the risk of death rapidly increased but at a slower rate compared to a subject with CKD.

Compared to other dyad states, the DM + IHD had the lowest risk of death at any given time, followed by $\mathrm{DM}+\mathrm{CKD}$. Subjects with IHD + CKD had a very high cumulative hazard of death, which was about six times higher compared with the corresponding values of the DM + IHD state at the 4th year. Furthermore, the hazard of death at IHD + CKD was far higher than the hazard of transitioning to DM + IHD + CKD state, showing that these individuals were less likely to move to the triad state. As noted, there was an elevated risk of death for DM + CKD subjects during the very early period compared with transitioning to $\mathrm{DM}+\mathrm{IHD}+\mathrm{CKD}$. For the entire system, we observed the highest risk of death by DM + IHD + CKD at any given time.

\section{Covariate effects on state occupation probabilities}

Table 1 presents the estimated odds ratios and 95\% confidence bands on state occupations for several covariates of interest such as age, gender, race/ethnicity, CCI, and dual eligibility. Note that a multistate system is characterized by state-to-state events and at-risk processes, which are both functions of time. Consequently, the covariate effect on the state occupation could vary over time. Therefore, we report the impact of covariate at a sequence of time points: at $365,730,1095$, and 1460 days corresponding to years $1,2,3$, and 4 . The estimated regression coefficients from the multistate model along with $p$-values are presented in Additional file 1: Table S2.

Age significantly affected the state occupation probabilities of all eight states and its effect was fairly uniform throughout the four time points for a given state, except for IHD and CKD. Interestingly, the direction of age effect appeared to be inconsistent among states. For example, older individuals had low odds of occupying the DM state or dyads and triad states with DM at any time. As expected, the odds of occupying the Death state for an older individual was relatively higher and the magnitude of effects due to age increment appeared to be the highest among all states. 

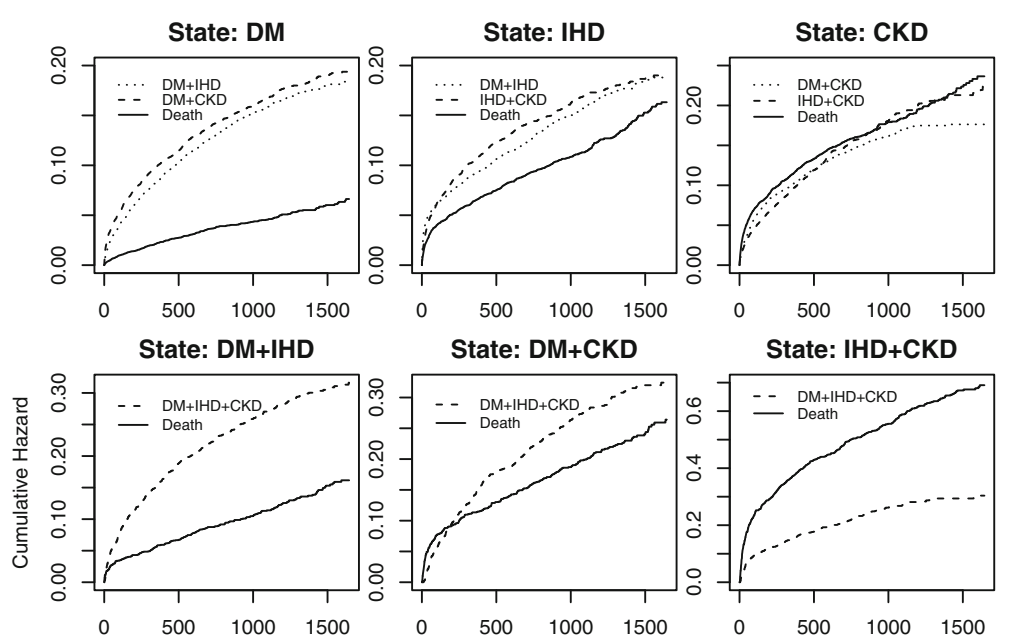

State: IHD+CKD

State: $\mathrm{DM}+\mathrm{IHD}+\mathrm{CKD}$

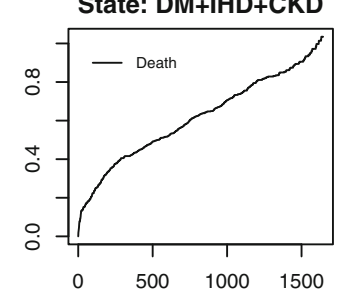

Days

Fig. 3 Estimated marginal cumulative state-to-state transition hazards for the multistate model. Note: different spans of y-axis were used for different states

Gender showed significant impacts on all states except IHD and DM + CKD. Clearly, the odds of occupying DM and CKD states for females were higher than males at any given time and the corresponding impact was relatively large for DM state. On the other hand, males had higher odds to be in $\mathrm{DM}+$ IHD, IHD + CKD, DM + IHD + CKD, and Death states at all the time points studied.

We evaluated the race/ethnicity difference on the state occupation probability, estimating odds ratios for other racial/ethnic groups, relative to White. The result suggested a fair consistency in racial/ethnic effects on the state occupation probability among Asian, NHPI and Other. Compared to Whites, for example, Asian, NHPI and Other had significantly higher odds to occupy DM, $\mathrm{DM}+\mathrm{CKD}, \mathrm{DM}+\mathrm{IHD}+\mathrm{CKD}$ states but low odds to be in IHD and IHD + CKD states at all four time points. Additionally, for NHPIs, there was a significantly high odds to be in DM + IHD and a low odds to be in Death state, compared to Whites.

The CCI appeared to be another important factor for state occupation in this multistate model. Higher values of CCI were associated with significantly higher odds for an individual to occupy the Death state at any given time. Subjects with higher CCI values were less likely to stay in DM, DM + IHD, DM + CKD, and DM + IHD + CKD states, compared to subjects with lower values. On the other hand, individuals with high CCI had higher odds to occupy the CKD state. Our results did not suggest any statistically significant associations between CCI and odds to be in IHD and IHD + CKD states.

Interestingly, the dual eligibility status did not show strong effects on the state occupation probabilities of the system except for DM and Death states. The dual eligibility factor reduced the odds to be in the DM state. However, an individual with the dual eligibility had significantly higher odds of occupying the Death state compared to non-eligible subjects, and the intensity of the effect increased over time.

\section{Covariate effects on cumulative transition hazards}

Table 2 summarizes the estimated hazard ratios for the 16 different cumulative state-to-state transitions and their corresponding 95\% point-wise confidence bands for the set of covariates (See Additional file 1: Table S3 for regression coefficients). The "From" and "To" columns of the table represent the type of transition and the rest of the columns provide relative cumulative hazards corresponding to each covariate. For example, the first cell under age variable shows that the relative $\mathrm{cu}$ mulative transition hazard from DM to DM + IHD was about 0.994 after one year due to a unit increment in age. Here, we focus our discussion comparing cumulative hazards at the beginning of the 4th year. 


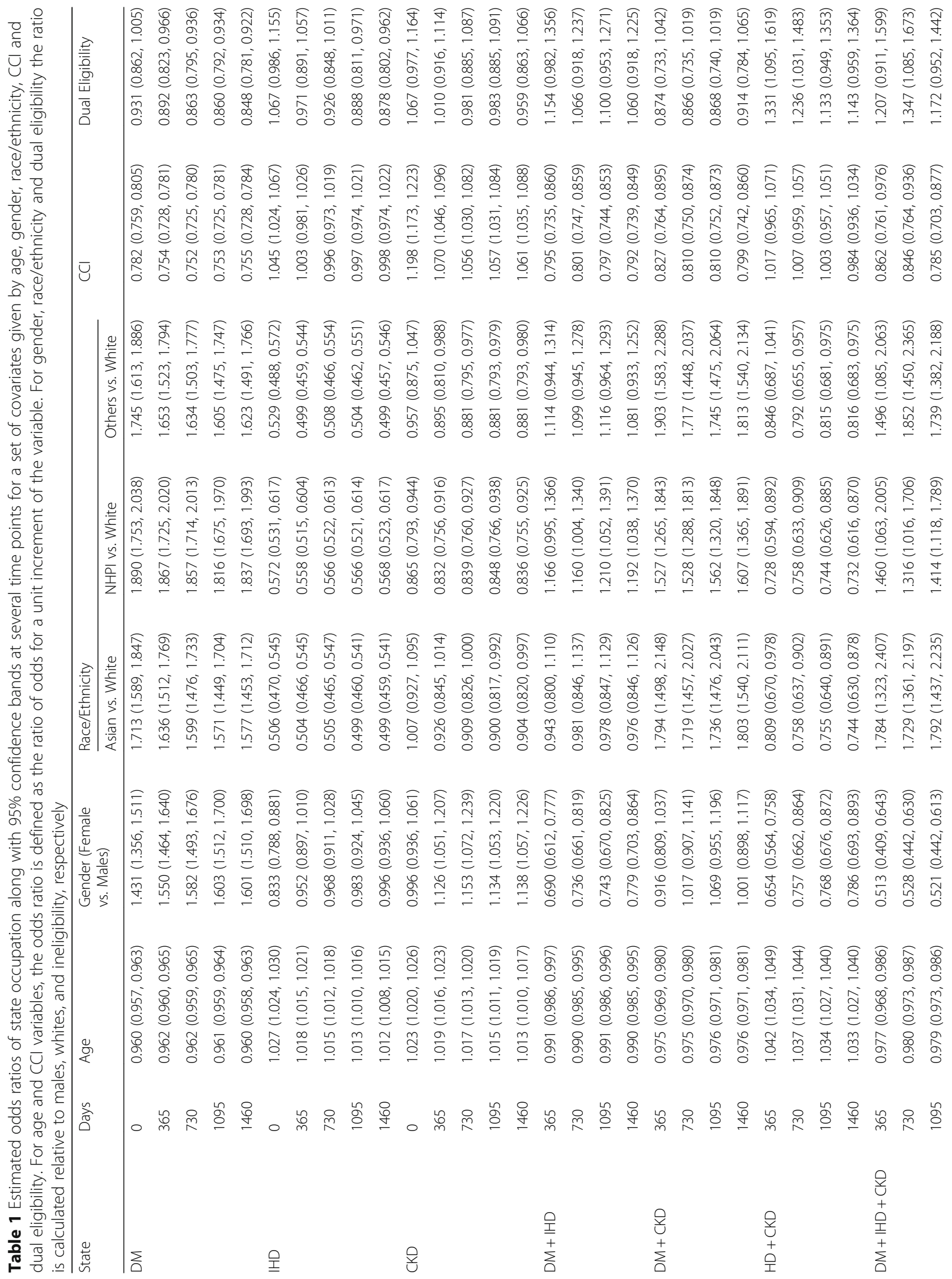




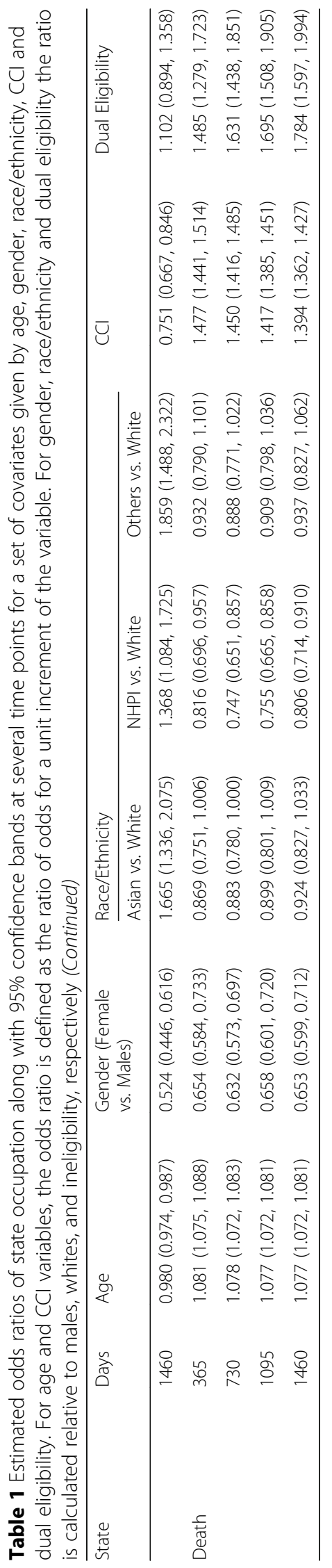




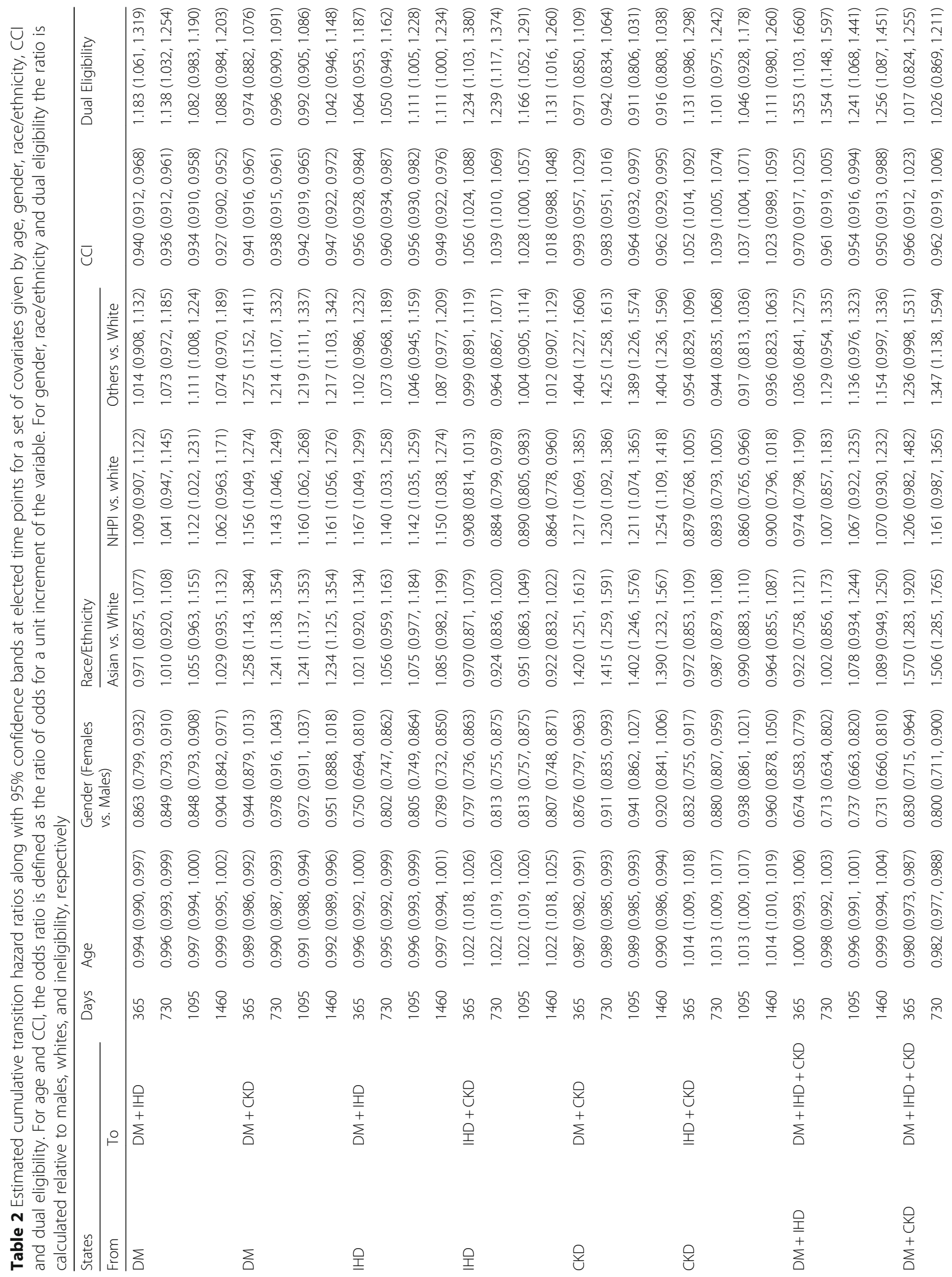









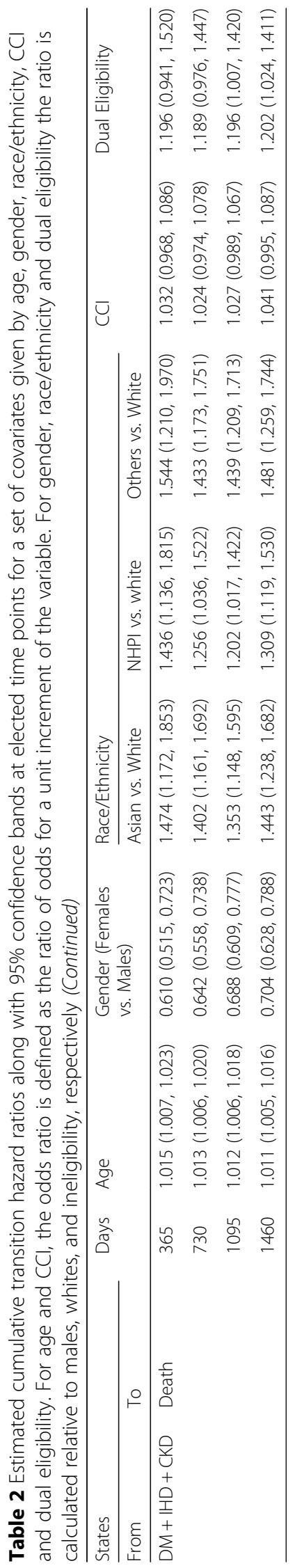


Age had a clear influence on the transition mechanism. Transitions that were directed towards Death state from IHD to IHD + CKD and from CKD to IHD + CKD showed significantly increased risks due to increase in age. However, age appeared to negatively affect the cumulative hazards of several transitions such as from DM to $\mathrm{DM}+\mathrm{CKD}$, from CKD to $\mathrm{DM}+\mathrm{CKD}$, and from $\mathrm{DM}$ $+\mathrm{CKD}$ to $\mathrm{DM}+\mathrm{IHD}+\mathrm{CKD}$, showing significantly lower cumulative hazards for older subjects.

As one of the interesting outcomes of this study, we found that males had a higher risk for transitioning between states compared to females, considering all types of transitions in the system. Among 16 transitions types, such differences were found to be significant for 12 cases.

Racial/ethnic discrepancies were found to affect the cumulative transition hazards between states. Compared to Whites, Asians had higher risks for moving from DM to $\mathrm{DM}+\mathrm{CKD}$, from CKD to $\mathrm{DM}+\mathrm{CKD}$, from $\mathrm{DM}+\mathrm{CKD}$ to $\mathrm{DM}+\mathrm{IHD}+\mathrm{CKD}$, from $\mathrm{IHD}+\mathrm{CKD}$ to $\mathrm{DM}+\mathrm{IHD}+$ CKD, from DM + CKD to Death, and from DM + IHD + CKD to Death. However, the relative risk of transferring from IHD state to Death was significantly lower for Asians. For NHPIs, transition hazards from DM to DM + CKD, from IHD to DM + IHD, from CKD to DM + CKD, from $\mathrm{DM}+\mathrm{CKD}$ to $\mathrm{DM}+\mathrm{IHD}+\mathrm{CKD}$, and from $\mathrm{DM}+$ $\mathrm{IHD}+\mathrm{CKD}$ to Death were relatively higher compared to Whites, but hazards from IHD to IHD + CKD and from IHD to Death were relatively lower for the NHPI group. The overall outcome of racial/ethnic differences seem to suggest that Whites had relatively lower risks for transferring from CKD to $\mathrm{DM}+\mathrm{CKD}$ and from $\mathrm{DM}+\mathrm{IHD}+$ CKD to Death, but they had an increased risk for transferring from IHD to Death compared to all other groups.

We observed that higher values of CCI were associated with increased risks of transitioning to the Death state. In several transitions such as from DM, IHD, CKD, and IHD + CKD states to Death, the increased risks were statistically significant. Furthermore, subjects with higher CCI were less likely to transition from DM to $\mathrm{DM}+\mathrm{IHD}$, from $\mathrm{DM}$ to $\mathrm{DM}+\mathrm{CKD}$, from IHD to $\mathrm{DM}+\mathrm{IHD}, \mathrm{CKD}$ to $\mathrm{DMI}+\mathrm{CKD}$, and from $\mathrm{DM}+\mathrm{CKD}$ to $\mathrm{DM}+\mathrm{IHD}+\mathrm{CKD}$.

Estimated relative risks for the dual eligibility suggested that it has a positive association with elderly subjects moving to the Death state. In fact, observed risks were significant for such transitions from all other states. Additionally, we found significantly increased risks associated with the dual eligibility on transitions from IHD to DM + IHD, from IHD to IHD + CKD, and from $\mathrm{DM}+\mathrm{IHD}$ to $\mathrm{DM}+\mathrm{IHD}+\mathrm{CKD}$.

\section{Discussion}

We investigated characteristics of a multistate system that was based on states defined by three major chronic conditions, DM, IHD, and CKD, for elderly individuals aged 65 and above, using five years of Hawaii Medicare data. In the interconnected disease network, an individual entered the system from DM, IHD, or CKD states and finally reaches the absorbing state Death following one of several possible paths.

During the five-year follow-up, we found that the probabilities of occupying the initial states continuously decrease, showing tendencies of individuals moving to the subsequent states. In particular, an individual with CKD had only about a $50 \%$ chance to stay in this initial state at the end of the five-year study period, declining the occupational probability at a faster rate compared to DM and IHD states. This could possibly be due to the serious co-morbidity risks associated with $\mathrm{CKD}$ condition. During this period, the state occupational probabilities at intermediate states almost reached stationary levels, and the probability of death continuously increased following a fairly linear trend with respect to time. The analysis of marginal transition hazards provides a clear picture of burdens of competing hazards on transitioning to various subsequent states initiating from a given state. For example, subjects with IHD + CKD had a greater risk of death than their risk of moving to $\mathrm{DM}+\mathrm{IHD}+\mathrm{CKD}$ state, at any given time. On the other hand, subjects with DM + IHD had a higher risk of moving to DM + IHD + CKD state than the corresponding risk of death, at any given time.

The results revealed several interesting covariate relationships on state occupational probabilities and transition hazards. We found that females had a relatively lower hazard for transitioning between states compared to males. It is a known fact that females have a longer lifespan compared to males [20,21]. Our results can possibly shed some light on this with respect to a chronic disease system. Among many racial/ethnic differences uncovered, we found that there was an increased risk for Asian and NHPI patients to transfer from $D M$ to $D M+C K D$ states while Whites had the highest risk of transferring from IHD to Death. Several other shared characteristics among Asians, NHPI, and Other groups compared to Whites were observed. Such racial/ethnic differences should be considered when healthcare providers/administrators develop early prevention strategies for subgroups of patients with higher risks.

We observed that older individuals were less likely to transfer from IHD to IHD + CKD, from CKD to DM + CKD, and from DM + CKD to DM + IHD + CKD. Also, individuals who had higher values of the CCI were less likely to transfer from several states to their subsequent states. Although some of these findings seem surprising, we want to draw attention to the magnitude of hazards 
by these factors on transferring directly to the Death state regardless of the magnitude of transitioning to subsequent clinical states, which were always high. We found that subjects with low economic status as reflected by their dual eligibility, had relatively high risks of death, which could possibly be due to living conditions, low income, or barriers to accessing healthcare.

Findings of this study can be strategically utilized and implemented in clinical and healthcare management settings to minimize mortality and transition risks among the elderly who are diagnosed with DM, IHD, and CKD conditions. We identified a wide range of covariate influences on state-to-state transition hazards. Subgroup-specific intervention plans can be established by considering the expected risks. For example, recommending periodic screenings or using tests with increased sensitivities in diagnosing diseases for high risk subgroups could provide a better chance for early detection or improved implementation of appropriate therapeutic strategies before those conditions worsen. High-risk subjects can be introduced to early prevention strategies or specialized care upon recommendations by their healthcare providers to minimize anticipated risks for comorbidities. Obviously, such approaches could result in extra cost. Hence, healthcare policymakers should evaluate these facts from a financial prospective, balancing the costs for required resources (including the patient care workforce for patients at more chronically ill stages) versus the cost of developing and implementing preventive strategies, as well as patient health and quality of life aspects.

There is a paucity of research literature exploring state-transition outcomes for chronic conditions including DM, IHD, and CKD within the general multistate framework. In contrast to ours, several studies utilizing multistate models focused on the progression of a given condition in different stages of the disease [22-26]. Other reports on DM, IHD, and CKD described risks of developing one condition given few other chronic conditions [27-31] and the burden of comorbidities among these patient populations [32-34]. In terms of survival, previous studies frequently focused on estimating hazards and mortality rates for DM, IHD, and CKD populations [35-37]. Interestingly, a study conducted using Third National Health and Nutritional Examination Survey revealed 10-year mortality rates as 7.7, 11.5, and $31.1 \%$ respectively for subjects without either diabetics or kidney disease, patients with diabetes but without kidney disease, and patients with both diabetes and kidney disease, demonstrating the devastating impact of co-occurring diabetic and kidney diseases [38]. This study also shed light on increased cardiovascular-related events rates for having both conditions together. Clearly, there is a lacking of research exploring the progressive patterns of DM, IHD, and CKD conditions and mortality in the multi-disease state-transition setting.

It is challenging and expensive to collect longitudinal data of a cohort of subjects that contains health status in a continuous time frame. However, health insurance databases are rich sources providing vital information which can be utilized in analyzing multistage disease transition systems similar to the model that we used. Compared to carefully collected follow-up data from a set of subjects for a few pre-selected conditions, health insurance data bases naturally provide access to a spectrum of diagnoses, which allows their widened applications to many public health problems.

Besides addressing the specific problem outlined based on diagnoses of DM, IHD, and CKD, a key interest of ours was to illustrate the use of health insurance data to evaluate survival characteristics of a chronic disease system. However, these approaches are not widely adopted yet. The large scale of the claims data, the intense effort needed to track subjects' transitions among multiple clinical stages, and data security issues, are just several reasons for its lack of popularity. The use of health insurance claims data may not be an ideal choice to evaluate disease systems as these databases are incapable of updating the health status of the subject efficiently in real time. The system only documents when a subject makes a claim. Due to a variety of reasons, there can be a considerable number of unreported incidents even though the specific events of interest have already occurred, such as unawareness of the event, use of multiple insurance plans, switching between plans, delayed or irregular hospital visits, and errors in reporting, etc. These issues could lead to underestimation in state-to-state hazard estimations. However, it is quite reasonable to assume that these issues are independent from subject-specific covariates in the general sense. The generalizability of outcomes to a wider population is a common potential limitation for most of the studies conducted based on claims data, as the inferences made could be specific to the population under investigation. Nevertheless, we believe that the use of longitudinal health insurance data provides an excellent opportunity to investigate healthcare problems.

There are several other limitations with this study. We determined individual's first disease occurrence history prior to 2009 using historical ICD-9 codes provided for several selected major conditions, but historical records of conditions can be limited and may not fully reflect the complete chronic conditions of the individual. This can possibly result in misidentification of subjects who had already diagnosed a chronic condition prior to 2009 as new cases, causing potential errors in survival estimates and in calculating the CCI. Furthermore, we 
found reporting errors exist in the claims data. We removed claims with dates found to be older than the date of death for several subjects. Although the proposed multistate model restricts individuals from entering into the system from intermediate states and transiting from initial states to the DM + IHD + CKD state, we observed such phenomenon for a handful of subjects in the data. However, to reduce the complexity, those beneficiaries were not included in the analysis. The maximum time period that these subjects were limited to was 5 years. To have a better understanding of such complex system, specifically in consideration of chronic diseases, a longer follow-up period is necessary. Due to the limited follow-up period the observed transitions subjected to right censoring. In fact, the right censoring rate in terms of an individual reaching to the final state was $87.2 \%$. It is important to note that our analysis did not consider temporal effects associated with the calendar time. For instance, we assumed a given state-to-state transition probability is independent of the calendar time. However, such transitions could potentially be influenced by time-varying factors. It is worthwhile to conduct future sensitivity analyses to evaluate the robustness of the results due to the impact of these time varying factors. Lastly, several other potential risk factors may not be considered in our multistate model. For example, we did not include obesity that can be interrelated and contribute to the development and progression of the chronic conditions. We did not include obesity as a covariate because the identification of obesity using diagnosis codes tends to be highly underestimated.

We believe this is the first study conducted investigating DM, IHD, and CKD together in the prospect of state transitions and survival in the elderly population. The research revealed many important covariate relationships with respect to state occupational probabilities and transitions. The results will be useful for healthcare providers and policymakers in prevention planning and implementation.

\section{Conclusion}

Using Hawaii Medicare database, we quantified survival characteristics of a multistate system that was defined by the occurrence of three chronic conditions (DM, IHD, and CKD) and their transition to the absorbing state Death, among individuals age 65 and above. Our study provides evidences that the clinical state transition mechanism could be subject- or subgroup-specific. Thus, consideration of subgroup-specific screening procedures and intervention plans for elderly individuals will be helpful for the optimal prevention and care of these chronic conditions.

\section{Additional file}

\begin{abstract}
Additional file 1: State occupation probabilities and transition hazards: A supplementary description of statistical methods used for estimating state occupation probabilities and transition hazards. Table S1. A summary matrix showing state-to-state transition counts for the chronic disease network. NA indicates transitions that are not applicable for the system. Table S2. Estimated regression coefficients that represent effects of covariates: age, gender, race/ethnicity, $\mathrm{CCl}$ and dual eligibility, on the state occupation probability at selected time points along with $\mathrm{p}$-values (in brackets). Table S3. Estimated regression coefficients that represent effects of covariates: age, gender, race/ethnicity, CCl and dual eligibility, on cumulative transition hazards at selected time points along with p-values (in brackets). Figure S1. Marginally estimated cumulative state-to-state transition hazards from state DM to subsequent states. Figure S2. Marginally estimated cumulative state-to-state transition hazards from state IHD to subsequent states along with bootstrap based 95\% point-wise confidence bands. Figure S3. Marginally estimated cumulative state-to-state transition hazards from state CKD to subsequent states along with bootstrap based 95\% point-wise confidence bands. Figure S4. Marginally estimated cumulative state-to-state transition hazards from state $\mathrm{DM}+\mathrm{HD}$ to subsequent states along with bootstrap based 95\% point-wise confidence bands. Figure S5. Marginally estimated cumulative state-to-state transition hazards from state DM+CKD to subsequent states along with bootstrap based $95 \%$ point-wise confidence bands. Figure S6. Marginally estimated cumulative state-to-state transition hazards from state IHD+CKD to subsequent states along with bootstrap based 95\% point-wise confidence bands. Figure S7. Marginally estimated state cumulative state-to-state transition hazards form state DM+IHD+CKD to Death state along with bootstrap based 95\% pointwise confidence bands. (PDF $406 \mathrm{~kb}$ )
\end{abstract}

\section{Abbreviations}

CCl: Charlson Comorbidity Index; CHD: Chronic Kidney Disease; DM: Diabetes mellitus; ICD-9: Classification of Disease 9th Revision; IHD: Ischemic Heart Disease

\section{Acknowledgments}

The authors would like to thank Munirih Taafaki for editing and proofreading the manuscript.

\section{Funding}

This work was partially supported grants U54MD007584 and U54MD007601 from National Institute on Minority Health and Health Disparities, and P20GM103466 and U54GM104944 from National Institute of General Medical Sciences (NIGMS), USA.

\section{Availability of data and materials}

The data set used for the study is not publicly available. Data will not be shared due to third party restrictions.

\section{Authors' contributions}

All authors involved with planning of the study. CS led the data analysis. All authors participated in the draft and revisions of the manuscript, and accepted the final draft

\section{Authors' information}

Chathura Siriwardhana, Ph.D. - Assistant Professor, Eunjung Lim, Ph.D. Assistant Professor, James Davis, Ph.D. - Associate Professor, and John J. Chen, Ph.D. - Professor \& Chair, Department of Complementary \& Integrative Medicine, University of Hawaii John A. Burns School of Medicine, Honolulu, HI 96813, USA.

\section{Ethics approval and consent to participate}

Ethics approval: The research protocol was approved by the Institutional Review Board at the University of Hawaii (CHS\#23362). Consent to participate: Not applicable, since the study was conducted as a retrospective study, using Hawaii Medicare health insurance claims database.

\section{Consent for publication}

Not applicable, since the study was conducted as a retrospective study, using Hawaii Medicare health insurance claims database. 


\section{Competing interests}

The authors declare that they have no competing interests.

\section{Publisher's Note}

Springer Nature remains neutral with regard to jurisdictional claims in published maps and institutional affiliations.

Received: 23 January 2018 Accepted: 10 June 2018

\section{Published online: 18 June 2018}

\section{References}

1. Murray $C L$, Lopez AD. Alternative projections of mortality and disability by cause 1990-2020: global burden of disease study. Lancet. 1997;349:1498-504

2. Jha V, Garcia-Garcia G, Iseki K, Li Z, Naicker S, Plattner B, Saran R, Wang AY, Yang CW. Chronic kidney disease: global dimension and perspectives. Lancet. 2013;382:260-72.

3. Naughton C, Bennett K, Feely J. Prevalence of chronic disease in the elderly based on a national pharmacy claims database. Age Ageing. 2006;35:633-6.

4. American Diabetes Association. Economic costs of diabetes in the U.S. in 2012. Diabetes Care. 2013;36(4):1033-46.

5. National Institute of Diabetes and Digestive and Kidney Diseases. Kidney Disease Statistics for the United States. https://www.niddk.nih.gov/-/media/ 88B73F5D3CF045EA8BC02A699842450B.ashx. Accessed on 26 Mar 2017.

6. Mozaffarian D, Benjamin EJ, Go AS, Arnett DK, Blaha MJ, et al. Heart Disease and Stroke Statistics-2016 Update, A Report From the American Heart Association. Circulation. 2015; https://doi.org/10.1161/CIR.0000000000000350.

7. Dieleman JL, Baral R, Birger M, Bui AL, Bulchis A, et al. US spending on personal health care and public health, 1996-2013. JAMA. 2016:316:2627-46.

8. Herzog CA, Asinger RW, Berger AK, Charytan DM, Díez J, et al. Cardiovascular disease in chronic kidney disease. A clinical update from kidney disease: improving global outcomes (KDIGO). Kidney Int. 2011; https://doi.org/10.1038/ki.2011.223.

9. Ahmed A, Campbell RC. Epidemiology of chronic kidney disease in heart failure. Heart Fail Clin. 2008:4:387-99.

10. Centers for Disease Control and Prevention. Prevalence of chronic kidney disease and associated risk factors-United States, 1999-2004. Morb Mortal Wkly Rep. 2007:56:161-5.

11. Coresh J, Astor BC, Greene T, Eknoyan G, Levey AS. Prevalence of chronic kidney disease and decreased kidney function in the adult US population: third national health and nutrition examination survey. Am J Kidney Dis. 2002:41:1-12.

12. Plantinga LC, Crews DC, Coresh J, Miller ER, Saran R, et al. Prevalence of chronic kidney disease in US adults with undiagnosed diabetes or prediabetes. Clin J Am Soc Nephrol. 2010; https://doi.org/10.2215/CJN.07891109.

13. Grundy SM, Benjamin IJ, Burke GL, Chait A, Eckel RH, et al. Diabetes and cardiovascular disease: a statement for healthcare professionals from the American Heart Association. Circulation. 1999;100:1134-46.

14. Vleming $L$, van der Pijl J, Lemkes HH, Westendorp RG, Maassen JA, et al. The DD genotype of the ACE gene polymorphism is associated with progression of diabetic nephropathy to end stage renal failure in IDDM. Clin Nephrol. 1999;51:133-40.

15. Ismail N, Becker B, Strzelczyk P, Ritz E. Renal disease and hypertension in non-insulin-dependent-diabetes mellitus. Kidney Int. 1999;55:1-28.

16. Centers for Disease Control and Prevention, International Classification of Diseases, Ninth Revision, Clinical Modification (ICD-9-CM). https://www.cdc gov/nchs/icd/icd9cm.htm. Accessed 26 Mar 2017.

17. Charlson ME, Pompei P, Ales KL, MacKenzie CR. A new method of classifying prognostic comorbidity in longitudinal studies: development and validation. J Chronic Dis. 1987:40:373-83.

18. Aalen $\mathrm{OO}$, Johansen S. An empirical transition matrix for nonhomogeneous Markov chains based censored observations. Scand J Stat. 1978:5:141-50.

19. Anderson PK, Klein JP. Regression analysis for multistate models based on a pseudo-value approach, with application to bone-marrow transplant studies. Scand J Stat. 2007:34:3-16.

20. Oeppen J, Vaupel JW. Broken Limits to Life Expectancy. Science. 2002;296: 1029-31.

21. Austad NS. Why women live longer than men: sex differences in longevity. Gend Med. 2006:3:79-92

22. Marshall G, Garg SK, Jackson WE, Holmes DL, Chase HP. Factors influencing the onset and progression of diabetic retinopathy in subjects with insulindependent diabetes mellitus. Ophthalmology. 1993;100(8):1133-9.
23. Stephens-Shields AJ, Spieker AJ, Anderson A, Drawz P, Fischer M, Sozio SM,

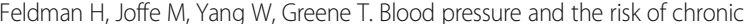
kidney disease progression using multistate marginal structural models in the CRIC study. Stat Med. 2017:36(26):4167-81. https://doi.org/10.1002/sim.7425.

24. Jonker JT, De Laet C, Franco OH, Peeters A, Mackenbach J, Nusselder WJ. Physical activity and life expectancy with and without diabetes: life table analysis of the Framingham heart study. Diabetes Care. 2006;29(1):38-43.

25. Peeters A, Bonneux L, Barendregt JJ, Mackenbach JP. Improvements in treatment of coronary heart disease and cessation of stroke mortality rate decline. Stroke. 2003;34:1610-4. https://doi.org/10.1161/01.STR.0000078661.72578.0A

26. Siannis F, Farewell VT, Head J. A multi-state model for joint modelling of terminal and non-terminal events with application to Whitehall II. Statist Med. 2007;26:426-42. https://doi.org/10.1002/sim.2342

27. Whaley-Connell AT, Sowers JR, McFarlane SI, Norris KC, Chen SC, Li S, Qiu Y, et al. Diabetes mellitus in CKD: kidney Early Evaluation Program (KEEP) and National Health and Nutrition and Examination Survey (NHANES) 1999-2004. Am J Kidney Dis. 2008;51(4 Suppl 2):S21-9.

28. Collins AJ, Foley RN, Gilbertson DT, Chen S. United States renal data system public health surveillance of chronic kidney disease and end-stage renal disease. Kidney Int Suppl. 2015;5:2-7.

29. Sarnak MJ, Levey AS, Schoolwerth AC, Coresh J, Culleton B, Hamm LL, et al. Kidney disease as a risk factor for development of cardiovascular disease. Hypertension. 2003:42:1050-65. https://doi.org/10.1161/01.HYP.0000102971.85504.7c.

30. Cai Q, Mukku VK, Ahmad M. Coronary artery disease in patients with chronic kidney disease: a clinical update. Curr Cardiol Rev. 2013;9(4):331-9.

31. Aronson D, Edelman ER. Coronary artery disease and diabetes mellitus. Cardiol Clin. 2014;32(3):439-55. https://doi.org/10.1016/j.ccl.2014.04.001.

32. Fraser SD, Roderick PJ, May CR, McIntyre N, Mclntyre C, Fluck RJ, et al. The burden of comorbidity in people with chronic kidney disease stage 3: a cohort study. BMC Nephrol. 2015;16:193. https:/doi.org/10.1186/s12882-015-0189-z.

33. Gruneir A, Markle-Reid M, Fisher K, Reimer H, Ma X, Ploeg J. Comorbidity burden and health services use in community-living older adults with diabetes mellitus: a retrospective cohort study. Can J Diabetes. 2016;40(1): 35-42. https://doi.org/10.1016/j.jcjd.2015.09.002.

34. Sachdev M, Sun JL, Tsiatis AA, Nelson CL, Mark DB, Jollis JG. The prognostic importance of comorbidity for mortality in patients with stable coronary artery disease. J Am Coll Cardiol. 2004:43(4):576-82.

35. Finegold JA, Asaria P, Francis DP. Mortality from ischaemic heart disease by country, region, and age: Statistics from World Health Organisation and United Nations. Int J Cardiol. 2013;168(2):934-45. https://doi.org/10.1016/j. ijcard.2012.10.046.

36. Eliasson M, Talbäck M, Rosén M. Improved survival in both men and women with diabetes between 1980 and 2004 - a cohort study in Sweden Cardiovasc Diabetol. 2008;7:32. https://doi.org/10.1186/1475-2840-7-32.

37. Tonelli M, Wiebe N, Culleton B, House A, Rabbat C, Fok M, McAlister F, Garg AX. Chronic kidney disease and mortality risk: a systematic review. J Am Soc Nephrol. 2006;17(7):2034-47.

38. Afkarian M, Sachs MC, Kestenbaum B, Hirsch IB, Tuttle KR, Himmelfarb J, de Boer $\mathrm{IH}$. Kidney disease and increased mortality risk in type 2 diabetes. J Am Soc Nephrol. 2013;24(2):302-8. https://doi.org/10.1681/ASN.2012070718.

\section{Ready to submit your research? Choose BMC and benefit from}

- fast, convenient online submission

- thorough peer review by experienced researchers in your field

- rapid publication on acceptance

- support for research data, including large and complex data types

- gold Open Access which fosters wider collaboration and increased citations

- maximum visibility for your research: over $100 \mathrm{M}$ website views per year

At BMC, research is always in progress.

Learn more biomedcentral.com/submissions 